\title{
Insulin-like growth factor binding protein-6 released from human mesenchymal stem cells confers neuronal protection through IGF-1R-mediated signaling
}

\author{
HYO-JIN JEON $^{1 *}$, JIHYE PARK ${ }^{1 *}$, JOO-HYUN SHIN ${ }^{1}$ and MI-SOOK CHANG ${ }^{1,2}$ \\ ${ }^{1}$ Laboratory of Stem Cell and Neurobiology, Department of Oral Anatomy, School of Dentistry and Dental Research Institute, \\ ${ }^{2}$ Neuroscience Research Institute, Seoul National University, Seoul 03080, Republic of Korea
}

Received June 9, 2017; Accepted October 4, 2017

DOI: $10.3892 / \mathrm{ijmm} .2017 .3173$

\begin{abstract}
Human bone marrow-derived mesenchymal stem cells (hMSCs) are a desirable cell source for cell-based therapy to treat nervous system injuries due to their ability to differentiate into specific cell types. In addition to their multipotency, hMSCs render the tissue microenvironment more favorable for tissue repair by secreting various growth factors. Our previous study demonstrated that hMSCs secrete several growth factors, including several insulin-like growth factor binding proteins (IGFBPs). Among these, IGFBP-6 binds with high affinity and inhibits insulin growth factor-2 (IGF-2) to inhibit the growth of IGF-2-dependent tumors. However, the function of IGFBP-6 in the nervous system remains to be fully elucidated. The present study investigated the protective effects of IGFBP-6 secreted by hMSCs on $\mathrm{H}_{2} \mathrm{O}_{2}$-injured primary cortical neuron cultures and lysolecithin-injured organotypic spinal cord slice cultures. Treatment of the $\mathrm{H}_{2} \mathrm{O}_{2}$-injured cortical neurons with conditioned media from hMSCs (hMSC-CM) increased the phosphorylation of Akt, reduced cell death and mitochondrial translocation of Bax, and regulated extracellular levels of IGF-1 and IGF-2. MTT assay, western blot analysis and ELISA were used to detect the cell viability and protein expression levels, respectively. An inhibitory antibody against IGFBP- 6 eliminated this hMSC-CM-mediated neuroprotective effect in the injured cortical neuron cultures and spinal cord slice cultures. In addition, treatment with cyclolignan picropodophyllin, an inhibitor of IGF-1 receptor (IGF-1R), significantly inhibited neuronal protection by hMSC-CM. These findings demon-
\end{abstract}

Correspondence to: Professor Mi-Sook Chang, Laboratory of Stem Cell and Neurobiology, Department of Oral Anatomy, School of Dentistry and Dental Research Institute, Seoul National University, 101 Daehak-ro, Jongno-gu, Seoul 03080, Republic of Korea E-mail: mschang@snu.ac.kr

${ }^{*}$ Contributed equally

Key words: cell death, growth factor, mesenchymal stem cells, neuroprotection, oxidative stress strated that hMSC-CM-mediated neuroprotection was attributed to IGF-1R-mediated signaling, potentiated via the inhibition of IGF-2 by IGFBP- 6 . The results of the present study provide insight into the mechanism by which hMSC administration may promote recovery from nerve injury.

\section{Introduction}

The reactive oxygen species (ROS) generated by oxidative stress in the central nervous system (CNS) contribute to the pathogenesis of neurodegenerative diseases, including Parkinson's disease, Alzheimer's disease and amyotrophic lateral sclerosis (1). Several effective factors have been reported to protect neurons and attenuate neuroinflammation against oxidative stress $(1,2)$. However, novel therapeutic strategies are required for the clinical application of neuroprotective factors.

Several previous studies have demonstrated a role for mesenchymal stem cells (MSCs) in promoting neuronal survival and the recovery of pathological symptoms in stroke, spinal cord injury and Parkinson's disease (3). In addition, experimental data have demonstrated that trophic factors released from MSCs have a therapeutic effect, termed a 'paracrine effect', promoting tissue repair, including neuronal survival, differentiation, axonal regeneration and endogenous angiogenesis (4).

In our previous study, the secretion of various growth factors from human MSCs (hMSCs) were analyzed, with vascular endothelial growth factor (VEGF), hepatocyte growth factor (HGF), stem cell factor (SCF) and members of the insulin-like growth factor binding protein (IGFBP) family observed. In addition, the expression of IGFBP-4 and - 6 was significantly enhanced in hMSC-conditioned medium (hMSC-CM) (5). A total of six distinct IGFBPs, designated IGFBP-1-6, function as carrier proteins for IGFs, and modulate IGF activities, including cell survival, proliferation, migration and differentiation in various cell types (6). IGFBPs are also important in biological functions by IGF-independent mechanisms (7). Despite their sequence homology, individual IGFBPs have distinct gene products and functional properties (8). IGFBP-6 is expressed in a variety of tissues, including the CNS, and its expression is developmentally regulated. In several cell lines, IGFBP-6 predominantly binds to IGF-2 and inhibits its function (9). 
However, the effect of IGFBP-6 on IGF-1 function remains to be fully elucidated, particularly in non-cancer cells. IGF-1 is a widely studied survival factor secreted from hMSCs and is involved in IGF-1 receptor (IGF-1R) signaling (10).

In the present study, a rat primary cortical neuron culture was used to examine the effect of IGFBP-6 released from hMSCs on neuronal cell death. The results suggested that IGFBP-6 was important in neuronal survival through activation of the Akt- and IGF-1R-mediated signaling pathway.

\section{Materials and methods}

hMSC culture. Cryopreserved adult bone marrow-derived hMSCs were purchased from Cambrex Bioscience (Walkersville, MD, USA). The hMSCs (passages 4-10) were cultured in Dulbecco's modified Eagle's medium (DMEM)-low glucose containing 10\% fetal bovine serum (FBS) (both from Gibco; Thermo Fisher Scientific, Inc., Waltham, MA, USA) at $37^{\circ} \mathrm{C}$ with $5 \% \mathrm{CO}_{2}$.

Primary cortical neuron-enriched cultures and $\mathrm{H}_{2} \mathrm{O}_{2}$ treatment. All procedures were performed with the approval of the Institutional Animal Care and Use Committee issued by Seoul National University (Seoul, Korea). Primary cortical neuron-enriched cultures were prepared from the cerebral cortices of E17-day-old Sprague-Dawley rat embryos. In brief, the cortical tissue was dissociated and cells were seeded in neurobasal-A medium (NB; Gibco; Thermo Fisher Scientific, Inc.) containing $10 \% \mathrm{FBS}$. After $24 \mathrm{~h}$, the medium was replaced with NB containing 2\% B27 (Gibco; Thermo Fisher Scientific, Inc.). These primary cortical neuron-enriched cultures were maintained for 7-10 days, with the medium replaced every 3 days. The primary cortical neurons were prepared in 24-well tissue culture plates for $\mathrm{H}_{2} \mathrm{O}_{2}$ treatment, based on a previously reported method (8), in serum-free NB containing $15 \mu \mathrm{M}$ $\mathrm{H}_{2} \mathrm{O}_{2}$ for 15 min (Fig. 1A). The medium was then replaced with serum-free NB or CM and cultured for $24 \mathrm{~h}$ at $37^{\circ} \mathrm{C}$.

hMSC-CM. The hMSCs $\left(5 \times 10^{4}\right.$ cells $\left./ 100 \mathrm{~mm} / \mathrm{dish}\right)$ were washed twice with phosphate-buffered saline (PBS; pH 7.4) and the medium was replaced with serum-free NB. After $18 \mathrm{~h}$, the medium was collected and added to the $\mathrm{H}_{2} \mathrm{O}_{2}$-treated primary cortical neuron-enriched cultures. For the IGFBP-6 inhibition experiment, $30 \mu \mathrm{g} / \mathrm{ml}$ of anti-IGFBP-6 antibody (cat. no. MAB8762; R\&D Systems, Inc., Minneapolis, MN, USA) was added for pre-incubation with the hMSC-CM for $30 \mathrm{~min}$ at $37^{\circ} \mathrm{C}(11)$.

PD98059, wortmannin and picropodophyllin treatment. The $\mathrm{H}_{2} \mathrm{O}_{2}$-treated primary cortical neuron-enriched cultures were pre-incubated with PD98059 $(50 \mu \mathrm{M}$; Promega Corp. Madison, WI, USA), wortmannin (100 nM; Sigma-Aldrich; Merck Millipore, Darmstadt, Germany), or picropodophyllin (PPP, 500 nM; Santa Cruz Biotechnology, Inc., Dallas, TX, USA) diluted in the hMSC-CM for 15,30 or $15 \mathrm{~min}$ at $37^{\circ} \mathrm{C}$, respectively (12-14). Following pre-incubation with the inhibitors, the cells were treated with $15 \mu \mathrm{M} \mathrm{H}_{2} \mathrm{O}_{2}$ for $15 \mathrm{~min}$.

Cell viability and apoptosis assays. Following treatment as described above, the viability of the cells was then analyzed using the 3-(4,5-dimethylthiaziazol-3-yl)-2,5-diphenyltetrazolium bromide (MTT) method. The cells were incubated with $1 \mathrm{mg} / \mathrm{ml} \mathrm{MTT} \mathrm{(Sigma;} \mathrm{Merck} \mathrm{Millipore)} \mathrm{for} 1 \mathrm{~h}$ at $37^{\circ} \mathrm{C}$. The medium was carefully aspirated, and dimethyl sulfoxide (DMSO) $(150 \mu \mathrm{l})$ was added to solubilize the colored formazan product. The optical density was read at $554 \mathrm{~nm}$. Terminal deoxynuceotidyl transferase dUTP nick-end labeling (TUNEL) staining was performed using the In Situ Cell Death Detection kit (Roche Diagnostics, Basel, Switzerland) according to the manufacturer's protocol. In the ventral region of the spinal cord slice cultures, the numbers of apoptotic cells were counted (magnification, x100). All images were captured using a confocal laser-scanning microscope (FV300; Olympus, Tokyo, Japan).

Immunoblotting. The primary cortical neuron-enriched cultures were washed twice with cold PBS and lysed with RIPA buffer containing $50 \mathrm{mM}$ Tris- $\mathrm{HCl}$ ( $\mathrm{pH} 7.4), 150 \mathrm{mM} \mathrm{NaCl}, 1 \% \mathrm{NP}-40$, $0.25 \%$ sodium deoxycholate, $0.2 \mathrm{mg} / \mathrm{ml}$ leupeptin, $0.2 \mathrm{mg} / \mathrm{ml}$ aprotinin, $0.1 \mathrm{M}$ phenylmethylsulfonylfluoride, $1 \mathrm{mM} \mathrm{Na} \mathrm{VO}_{4}$ and $0.5 \mathrm{M} \mathrm{NaF}$. The lysates were centrifuged at $13,500 \mathrm{x} \mathrm{g}$ for $15 \mathrm{~min}$ at $4^{\circ} \mathrm{C}$, and $30 \mu \mathrm{g}$ of the supernatants were loaded onto $12 \%$ sodium dodecyl sulfate-polyacrylamide gel electrophoresis (SDS-PAGE) gels. The membrane was blocked in Tris-buffered saline containing $3 \%$ bovine serum albumin (BSA) for $1 \mathrm{~h}$ at room temperature. It was incubated overnight at $4{ }^{\circ} \mathrm{C}$ with the following primary antibodies: Anti-Akt (1:1,000; cat. no. 9272S), anti-phosphorylated (p)Akt (1:1,000; cat. no. 4058S) (both from Cell Signaling Technology, Inc., Beverly, MA, USA), anti-B-cell lymphoma 2-like protein 4 (Bax; 1:1,000; cat. no. 556467; BD Pharmingen, San Diego, CA, USA), anti-cyclooxygenase (COX) IV (1:1,000; cat. no. 4644S; Cell Signaling Technology, Inc.), and anti- $\alpha$-tubulin (1:5,000; cat. no. T5168; Sigma-Aldrich; Merck Millipore). The primary antibodies were visualized using a chemiluminescence detection system (Thermo Fisher Scientific, Inc.) after incubation with goat anti-rabbit or anti-mouse horseradish peroxidase-conjugated secondary antibodies (cat. nos. A0545 and A2554; Sigma-Aldrich; Merck Millipore) for $1 \mathrm{~h}$ at room temperature. Mitochondrial and cytosolic fractions were isolated with the Mitochondrial Fraction kit (Active Motif, Carlsbad, CA, USA) for Bax analysis. ImageJ software 1.6.0 version (NIH, Bethesda, MD, USA) was used for quantification of immunoblotting.

Enzyme-linked immunosorbent assay (ELISA) for IGF-1 and $I G F-2$. The primary rat cortical neurons in $60-\mathrm{mm}$ dishes were cultured in serum-free base medium with the additional treatment according to the experimental groups described above. After $30 \mathrm{~h}$, the supernatant was collected and centrifuged at $135 \mathrm{x} \mathrm{g}$ for $5 \mathrm{~min}$ at room temperature. The concentrations of IGF-1 or IGF-2 in the supernatant were quantified using a rat IGF-1 ELISA kit or a rat IGF-2 ELISA kit (cat. nos. ab213902 and ab213903; Abcam), respectively, according to the manufacturer's protocols. The optical density at $450 \mathrm{~nm}$ was measured using a microplate reader (Epoch2; Bio-Tek Instruments, Inc., Winooski, VT, USA).

Organotypic spinal cord slice culture. The organotypic slice cultures were generated as previously described (15). Briefly, the lumbar spinal cords of 16-day-old post-natal Sprague-Dawley rats were removed after euthanizing with $\mathrm{CO}_{2}$ gas. Nerve 

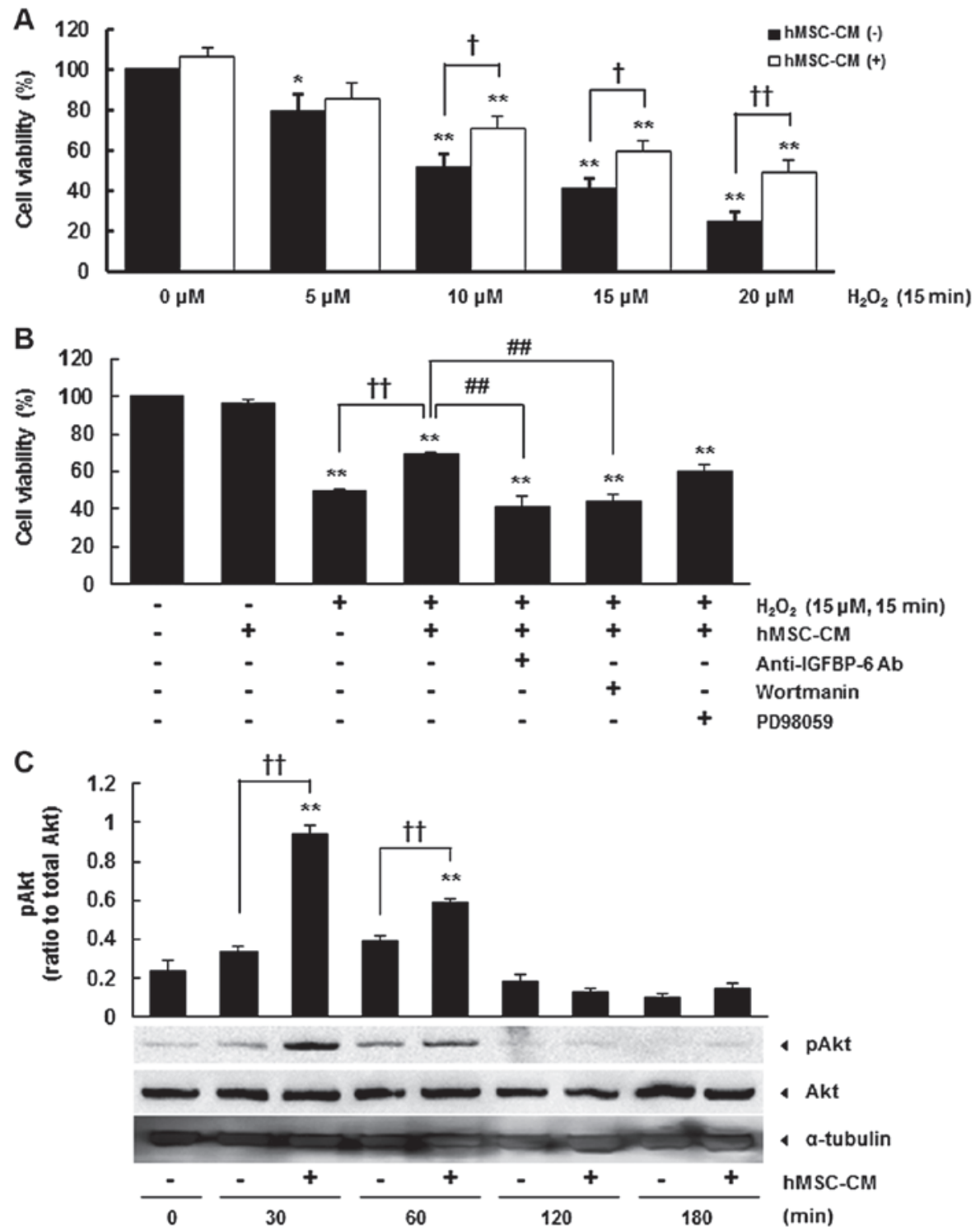

Figure 1. Neuroprotective effect of hMSC-CM is attributed to the release of IGFBP-6 and activation of Akt in $\mathrm{H}_{2} \mathrm{O}_{2}$-treated primary cortical neurons. (A) Primary cortical neurons were treated with $\mathrm{H}_{2} \mathrm{O}_{2}(0-20 \mu \mathrm{M})$ for 15 min and then incubated with or without hMSC-CM. (B) Following $\mathrm{H}_{2} \mathrm{O}_{2}$ treatment (15 $\mu \mathrm{M} \mathrm{H}_{2} \mathrm{O}_{2}$ for $15 \mathrm{~min}$ ), the cells were incubated with hMSC-CM or hMSC-CM pretreated with anti-IGFBP-6-Ab. The cells were also pre-incubated with PD98059 $(50 \mu \mathrm{M})$ or wortmannin $(100 \mathrm{nM})$ prior to $\mathrm{H}_{2} \mathrm{O}_{2}$ treatment and incubation with hMSC-CM. All 3-(4,5-dimethylthiaziazol-3-yl)-2,5-diphenyl tetrazolium bromide assays were performed $24 \mathrm{~h}$ following $\mathrm{H}_{2} \mathrm{O}_{2}$ treatment. (C) Expression levels of pAkt in $\mathrm{H}_{2} \mathrm{O}_{2}$-treated primary cortical neurons were observed 30,60,120 and 180 min following hMSC-CM treatment by immunoblotting. The data are presented as the mean \pm standard error of the mean of at least four independent experiments. " $\mathrm{P}<0.05$ and ${ }^{* *} \mathrm{P}<0.01$ vs. control; ${ }^{\dagger} \mathrm{P}<0.05$ and ${ }^{\dagger+} \mathrm{P}<0.01$ vs. $\mathrm{H}_{2} \mathrm{O}_{2}(-)$ hMSC-CM; ${ }^{\# \#} \mathrm{P}<0.01$ vs. $\mathrm{H}_{2} \mathrm{O}_{2}(+)$ hMSC-CM. Analysis of variance followed by the Newman-Keuls post hoc test were used. hMSC-CM, human mesenchymal stem cell-conditioned medium; pAKT, phosphorylated Akt; IGFBP-6 Ab, insulin-like growth factor binding protein 6 antibody.

roots and excess connective tissue were then collected in cold Hank's balanced salt solution (Gibco; Thermo Fisher Scientific, Inc.) containing $6.4 \mathrm{mg} / \mathrm{ml}$ glucose. Using a Mcllwain tissue chopper (Mickle Laboratory Engineering, Gomshall, UK), the spinal cords were finely cut, and four slices were carefully placed and co-cultured on a membrane insert (Millicell-CM; EMD Millipore, Billerica, MA, USA) for 7 days at $37^{\circ} \mathrm{C}$ in a 6 -well plate with $1 \mathrm{ml}$ of culture media containing 50\% Eagle's minimum essential medium (Gibco; Thermo Fisher Scientific, Inc.), $6.4 \mathrm{mg} / \mathrm{ml}$ glucose and $20 \mathrm{mM}$ HEPES (Sigma; Merck Millipore). The media was replaced twice a week.

Demyelination of organotypic spinal cord slices and hMSC transplantation. At 7 days following the generation of the spinal cord slice cultures, the slices were treated with $0.5 \mathrm{mg} / \mathrm{ml}$ of lysolecithin (LPC; Sigma; Merck Millipore) for $17 \mathrm{~h}$ at $37^{\circ} \mathrm{C}$ to induce demyelination, as previously described (15). The media was then replaced with fresh media, with or without anti-IGFBP- 6 antibody $(30 \mu \mathrm{g} / \mathrm{ml})$. The hMSCs $\left(3 \times 10^{4}\right.$ cells $\left./ 2.5 \mu \mathrm{l}\right)$ were transplanted into the ventral region of the spinal cord slice cultures using aspirator tube assemblies for microcapillary pipettes (Sigma; Merck Millipore) and the cultures were incubated for 1 week.

Statistical analysis. Statistical analysis was performed using the language R (R Development Core Team 2010) and data were and evaluated using one-way analysis of variance (ANOVA) analysis of variance followed by the Newman-Keuls post hoc test. $P<0.05$ 
A
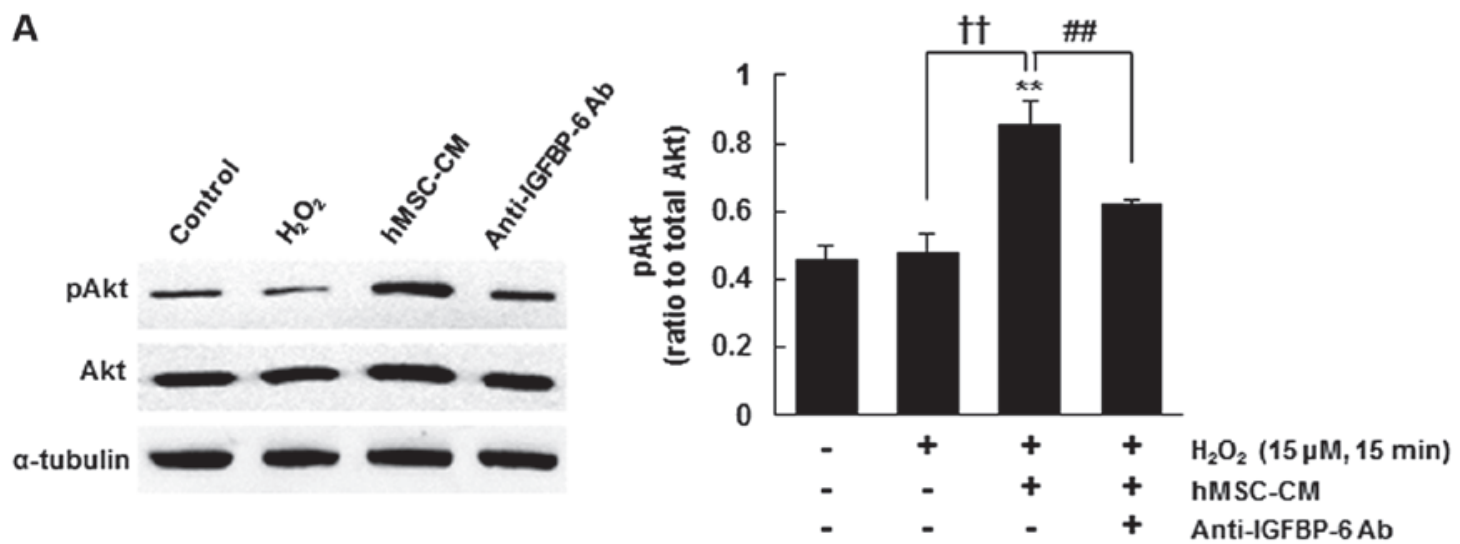

B
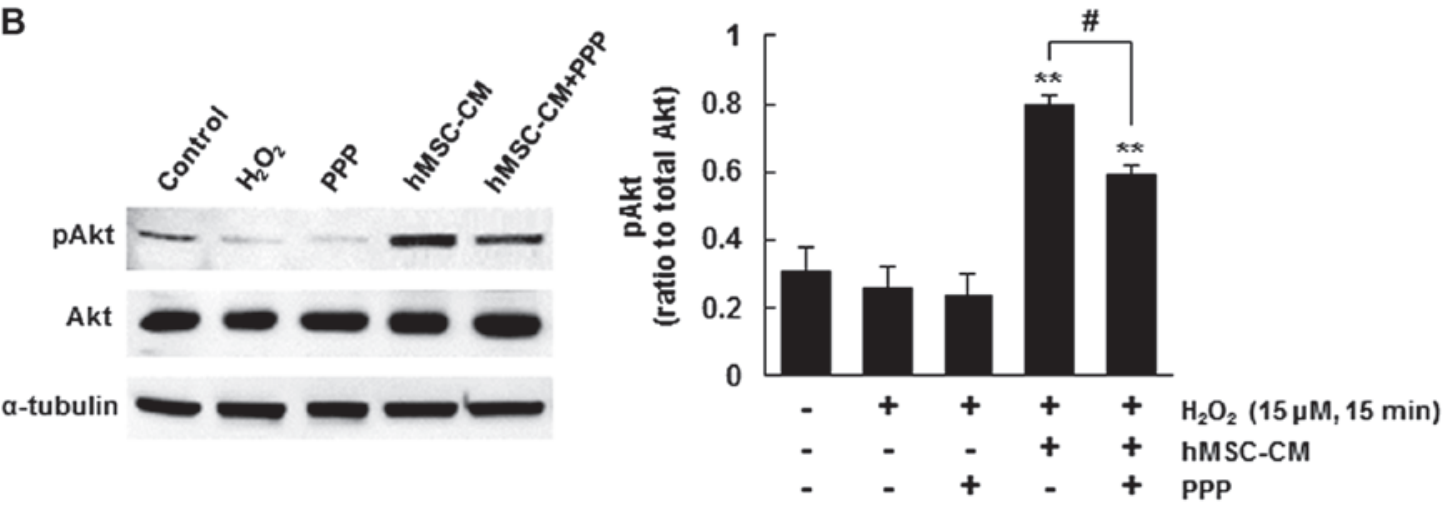

Figure 2. IGFBP-6 activates Akt in oxidative stress-damaged primary cortical neurons via IGF-1 receptor-dependent signaling. (A) Phosphorylation of Akt was examined in primary cortical neurons incubated with hMSC-CM or hMSC-CM pretreated with an anti-IGFPB-6-Ab following $\mathrm{H}_{2} \mathrm{O}_{2}$ treatment. (B) Primary cortical neurons were pre-incubated with PPP (500 nM) for 15 min prior to $\mathrm{H}_{2} \mathrm{O}_{2}$ treatment. Expression levels of pAkt were detected 30 min following $\mathrm{H}_{2} \mathrm{O}_{2}$ treatment. Data are presented as the mean \pm standard error of the mean of at least four independent experiments. ${ }^{* *} \mathrm{P}<0.01$, vs. control; ${ }^{\dagger} \mathrm{P}<0.01$ vs. $\mathrm{H}_{2} \mathrm{O}_{2}(-)$ hMSC-CM; ${ }^{\#} \mathrm{P}<0.05$ and ${ }^{\# \#} \mathrm{P}<0.01$ vs. $\mathrm{H}_{2} \mathrm{O}_{2}(+)$ hMSC-CM. Analysis of variance followed by the Newman-Keuls post hoc test were used. hMSC-CM, human mesenchymal stem cell-conditioned medium; pAKT, phosphorylated Akt; IGFBP-6 Ab, insulin-like growth factor binding protein 6 antibody; PPP, picropodophyllin.

was considered to indicate a statistically significant difference. All data are presented as the mean \pm standard error of the mean.

\section{Results}

Neuroprotective hMSC-CM signals by IGFBP-6 through the activation of Akt in $\mathrm{H}_{2} \mathrm{O}_{2}$-treated primary neurons. The present study examined the neuroprotective effect of hMSC-CM using primary cortical neuron cultures damaged using various concentrations of $\mathrm{H}_{2} \mathrm{O}_{2}(5-20 \mu \mathrm{M}$ for $15 \mathrm{~min})$, and found that hMSC-CM significantly increased cell survival of the cortical neurons exposed to $\mathrm{H}_{2} \mathrm{O}_{2}$ concentrations of 10,15 and $20 \mu \mathrm{M}$ (Fig. 1A). The concentration of $15 \mu \mathrm{M}$ $\mathrm{H}_{2} \mathrm{O}_{2}$ was selected to induce cell death of cortical neurons in subsequent experiments as this concentration resulted in $40.7 \pm 4.6 \%$ cell death (Fig. 1A). In our previous study, it was reported that transplanted hMSCs protected against cell death in injured slice cultures and one of the growth factors secreted by hMSCs was IGFBP-6 (5). Therefore, the present study aimed to determine whether IGFBP-6 was responsible for the hMSC-CM-mediated neuronal protection. Of note, the neutralizing antibody against IGFBP- 6 eliminated the hMSC-CM-mediated neuronal protection and decreased cell viability to the level found in the $\mathrm{H}_{2} \mathrm{O}_{2}$-treated culture $(\mathrm{P}<0.01)$ (Fig. 1B). These results indicated that IGFBP-6 was crucial in the neuroprotection mediated by hMSC-CM.
To identify the signal transduction pathway responsible for mediating neuroprotection by IGFBP-6 in hMSC-CM, the present study examined the activation of various kinases known to be involved in IGF signaling in primary cortical neurons. Wortmannin, a pharmacological inhibitor of the phosphoinositide 3-kinase (PI3K)/Akt pathway, suppressed the hMSC-CM-mediated neuroprotection of damaged primary cortical neurons $(\mathrm{P}<0.01)$ (Fig. 1B), as did anti-IGFBP-6 antibody, which confirmed the importance of Akt. However, PD98059, a pharmacological inhibitor of extracellular signal-regulated kinase (ERK), did not affect the neuroprotection induced by hMSC-CM (Fig. 1B). A significant increase in pAkt was observed in the hMSC-CM-treated cells $30 \mathrm{~min}$ and $1 \mathrm{~h}$ following hMSC-CM treatment, compared with that in the controls under oxidative stress $(\mathrm{P}<0.01)$ (Fig. $1 \mathrm{C})$. These results suggested that the Akt signaling pathway was important in IGFBP-6-mediated neuroprotection in primary cortical neurons.

Akt is activated by IGFBP-6 via IGF-1R. The neuroprotective IGFBP-6 signaling pathway may be associated with the activation of Akt (Fig. 1B and C). To confirm this, the phosphorylation level of Akt in hMSC-CM was determined following $\mathrm{H}_{2} \mathrm{O}_{2}$ treatment, with or without anti-IGFBP-6 antibody incubation. The protein level of pAkt was significantly increased in the hMSC-CM-treated primary cortical neurons following the induction of oxidative stress $(\mathrm{P}<0.01)$ (Fig. 2A). However, 

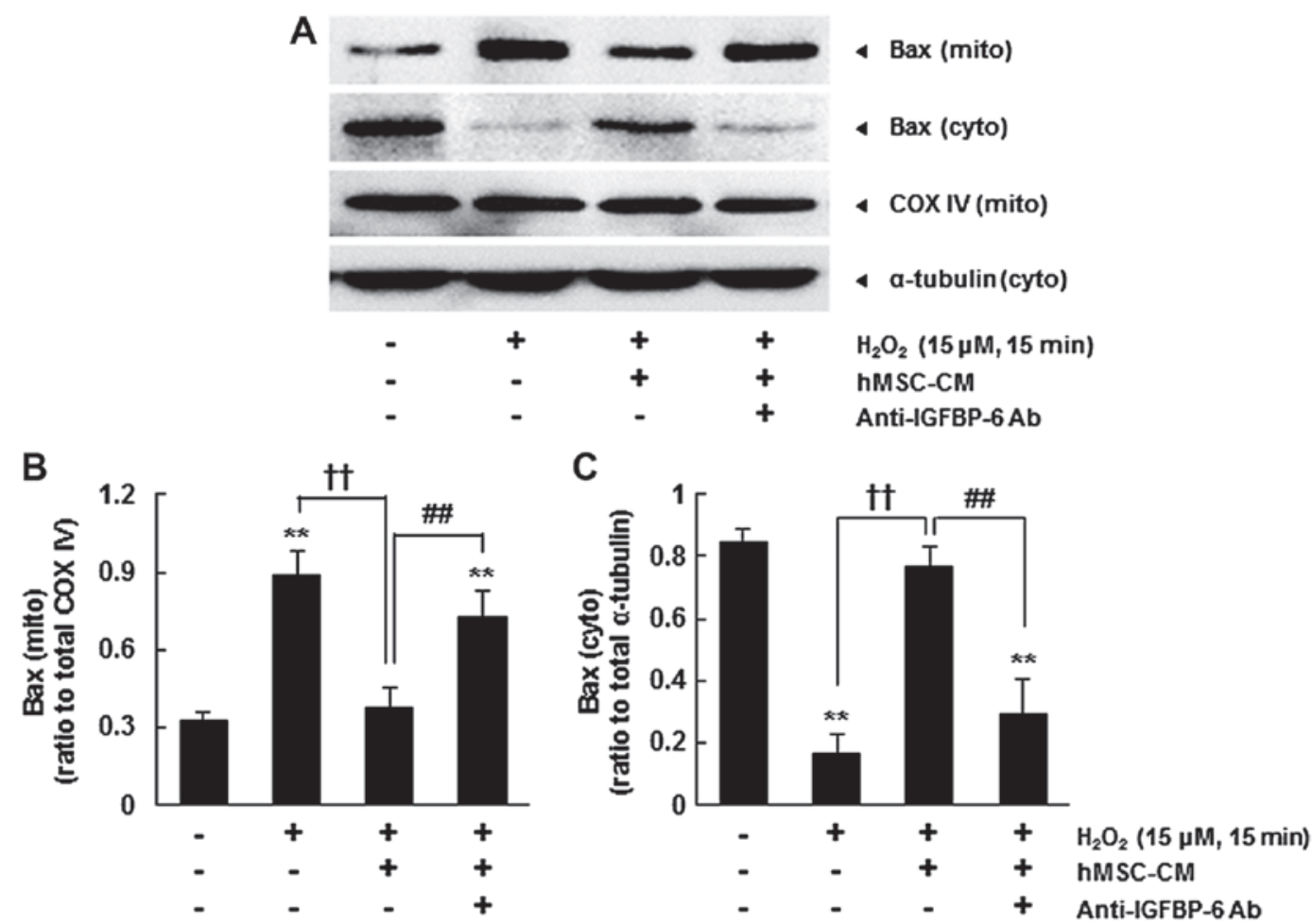

Figure 3. IGFBP-6 modulates the translocation of Bax in primary cortical neurons. (A) Immunoblotting and quantification of cytosolic and mitochondrial protein levels of Bax in primary cortical neurons incubated with hMSC-CM or hMSC-CM pretreated with anti-IGFPB-6 antibody following $\mathrm{H}_{2} \mathrm{O}_{2}$ treatment. (B) Mitochondrial and (C) cytosolic levels of Bax were quantified $24 \mathrm{~h}$ following $\mathrm{H}_{2} \mathrm{O}_{2}$ treatment. The data are presented the mean \pm standard error of the mean of at least four independent experiments. ${ }^{* *} \mathrm{P}<0.01$ vs. control; ${ }^{\dagger} \mathrm{P}<0.01$ vs. $\mathrm{H}_{2} \mathrm{O}_{2} ;{ }^{\# \#} \mathrm{P}<0.01 \mathrm{vs}$. hMSC-CM. Analysis of variance followed by the Newman-Keuls post hoc test were used. hMSC-CM, human mesenchymal stem cell-conditioned medium; cyto, cytosolic; mito, mitochondrial; Bax, B-cell lymphoma 2-like protein 4; COX-IV, cyclooxygenase IV; IGFBP-6 Ab, insulin-like growth factor binding protein-6 antibody.

pre-incubation with the anti-IGFBP-6 antibody eliminated the hMSC-CM-mediated activation of Akt and decreased the level of pAkt to the level observed in the $\mathrm{H}_{2} \mathrm{O}_{2}$-treated condition cultures $(\mathrm{P}<0.01)$ (Fig. 2A). Several studies have reported that the activation of Akt is involved in the IGF-1R downstream pathway (16). Using PPP, an inhibitor of IGF-1R, it was showed that the neuroprotective effect of hMSC-CM occurred through the IGF-1R-mediated activation of Akt. PPP treatment significantly reduced the hMSC-CM-mediated phosphorylation of Akt $(\mathrm{P}<0.05)$ (Fig. 2B). These results suggested that hMSC-CM protected the primary cortical neurons against oxidative stress-induced cell death through the activation of IGF-1R by IGFBP-6.

Modulation of Bax translocation by IGFBP-6. IGF-induced survival effects are regulated by Akt signaling, which interrupts the mitochondria-mediated apoptotic pathway and translocation of Bax, a regulator of apoptosis (17). The majority of Bax present in the cytosol translocates into the mitochondrial membrane during apoptosis $(18,19)$. Oxidative stress induced by $\mathrm{H}_{2} \mathrm{O}_{2}$ treatment significantly increased the level of Bax in the mitochondria ( 2.8-fold vs. control) and significantly reduced the cytosolic level of Bax $(\mathrm{P}<0.01)$ (Fig. 3). By contrast, hMSC-CM treatment inhibited the translocation of Bax and significantly reduced the level of mitochondrial Bax induced by $\mathrm{H}_{2} \mathrm{O}_{2}$ to the control level, restoring the cytosolic level of Bax. However, pre-incubation with the anti-IGFBP-6 antibody reversed the effect of hMSC-CM treatment and increased the mitochondrial level of Bax following $\mathrm{H}_{2} \mathrm{O}_{2}$ expo- sure ( 1.9-fold vs. control), whereas the cytosolic level of Bax was reduced $(\mathrm{P}<0.01)$ (Fig. 3). These results suggested that the IGFBP-6-induced activation of Akt inhibited the translocation of endogenous Bax from the cytoplasm to mitochondria, thereby promoting neuronal survival.

Extracellular levels of IGF-1 and IGF-2 by IGFBP-6 in $\mathrm{H}_{2} \mathrm{O}_{2}$-treated primary cortical neurons. As shown in Fig. 2B, the neuroprotective effect of IGFBP- 6 was regulated by IGF-1R signaling. However, IGFBP-6 has a higher binding affinity for IGF-2 than for IGF-1 and inhibits IGF-2 (9). Therefore, ELISA was used in the present study to examine the extracellular levels of IGF-1 or IGF-2 in damaged primary cortical neurons, with or without IGFBP-6, in hMSC-CM. Oxidative stress significantly increased the extracellular level of IGF-2, but not that of IGF-1, in the primary cortical neurons $(\mathrm{P}<0.01)$ (Fig. 4). By contrast, hMSC-CM treatment significantly increased the extracellular level of IGF-1 ( $\mathrm{P}<0.05)$ (Fig. 4A), and reversed the higher level of extracellular IGF-2 induced by $\mathrm{H}_{2} \mathrm{O}_{2}$ to the control level $(\mathrm{P}<0.01)$ (Fig. 4B). Treatment with hMSC-CM but without IGFBP-6 also significantly increased the extracellular level of IGF-1, compared with that in the control $(\mathrm{P}<0.05)$ (Fig. 4A). However, the enhanced level of extracellular IGF-2 in the cortical neurons damaged by $\mathrm{H}_{2} \mathrm{O}_{2}$ was maintained, even when treated with hMSC-CM without IGFBP-6 (P<0.05) (Fig. 4B). These results indicated that IGFBP- 6 significantly inhibited the oxidative stress-induced increase in extracellular levels of IGF-2, but did not directly regulate the extracellular levels of IGF-1. 

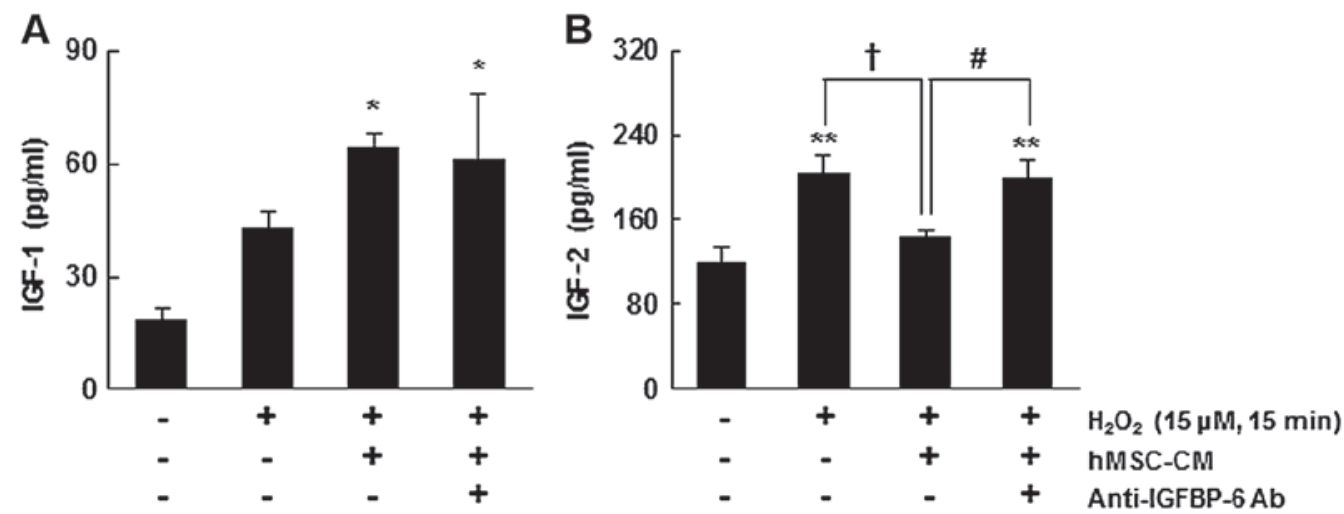

Figure 4. IGFBP-6 inhibits extracellular IGF-2 induced by oxidative stress in primary cortical neurons. Concentrations of (A) IGF-1 and (B) IGF-2 in the supernatant of primary cortical neuron cultures incubated with hMSC-CM or hMSC-CM pretreated with anti-IGFPB-6-Ab following $\mathrm{H}_{2} \mathrm{O}_{2}$ treatment were analyzed using an enzyme-linked immunosorbent assay. The data are presented the mean \pm standard error of the mean of at least four independent experiments. "P<0.05 and ${ }^{* *} \mathrm{P}<0.01$ vs. control; ${ }^{\dagger} \mathrm{P}<0.05$ vs. $\mathrm{H}_{2} \mathrm{O}_{2} ;{ }^{;} \mathrm{P}<0.05$ vs. hMSC-CM. Analysis of variance followed by the Newman-Keuls post hoc test were used. hMSC-CM, human mesenchymal stem cell-conditioned medium; IGF, insulin-like growth factor; IGFBP-6 Ab, insulin-like growth factor binding protein-6 antibody.
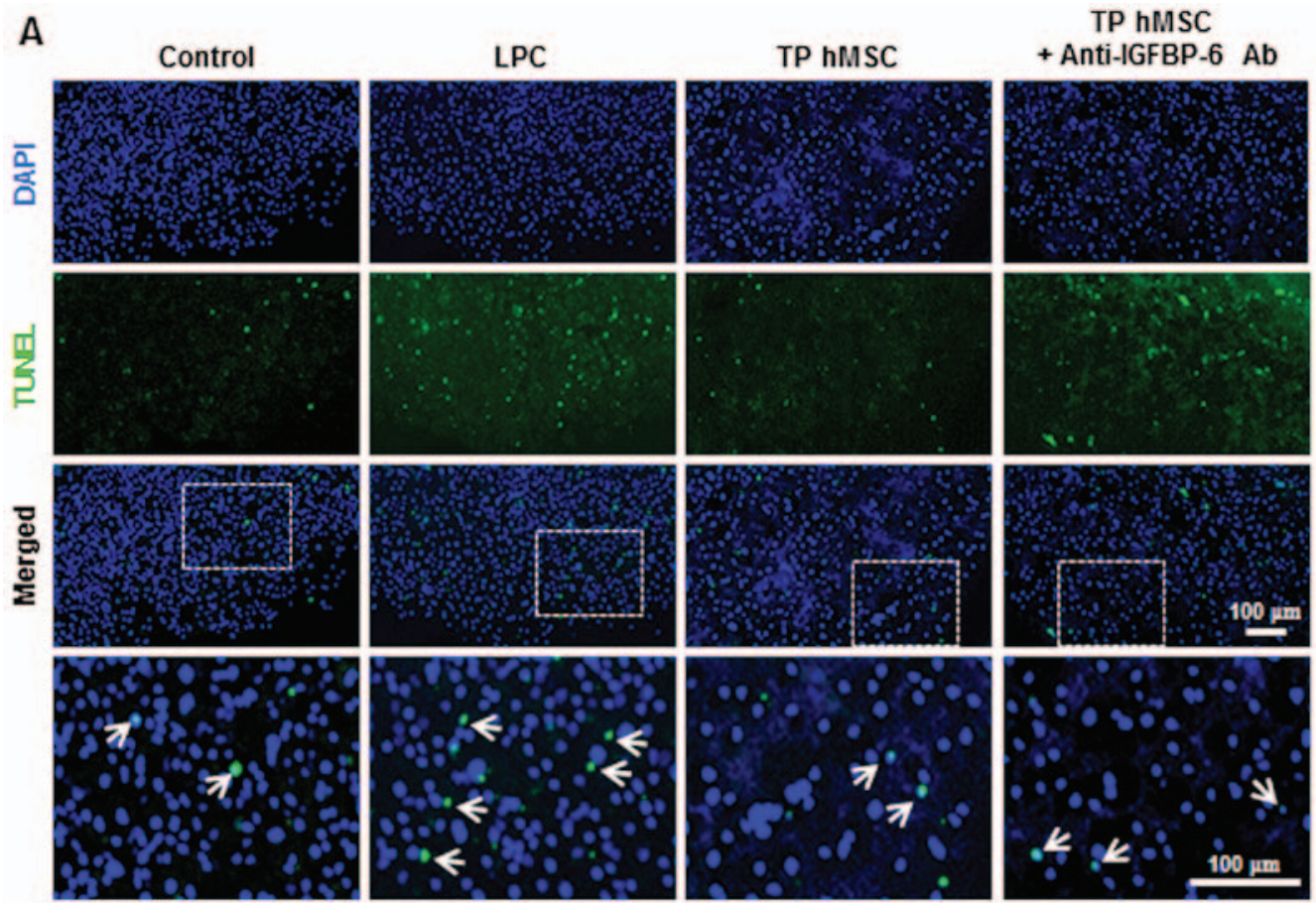

B

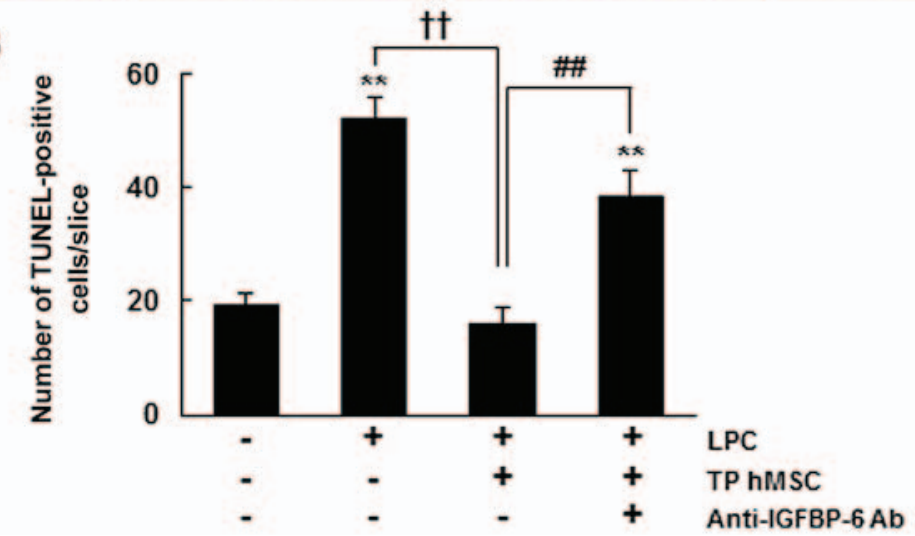

Figure 5. Neuroprotective effect of hMSCs is attributed to the release of IGFBP-6 in LPC-treated organotypic spinal cord slice cultures. (A) hMSCs or hMSCs incubated with anti-IGFBP-6-Ab were transplanted into LPC-treated spinal cord slice cultures. Scale bar, $100 \mu \mathrm{m}$ (B) Cell death was examined 7 days following LPC treatment by TUNEL staining. Arrows indicate fluorescence staining with TUNEL. The number of TUNEL-positive cells was quantified as the mean \pm standard error of the mean of three independent experiments. ${ }^{* *} \mathrm{P}<0.01$ vs. control; ${ }^{\dagger \dagger} \mathrm{P}<0.01 \mathrm{vs}$. LPC treatment; ${ }^{\# \#} \mathrm{P}<0.01$ vs. transplantation of hMSCs. Analysis of variance followed by the Newman-Keuls post hoc test were used. hMSC-CM, human mesenchymal stem cell-conditioned medium; LPC, lysolecithin; IGFBP-6 Ab, insulin-like growth factor binding protein 6 antibody; TUNEL, terminal deoxynuceotidyl transferase dUTP nick-end labeling; TP, transplantation. 


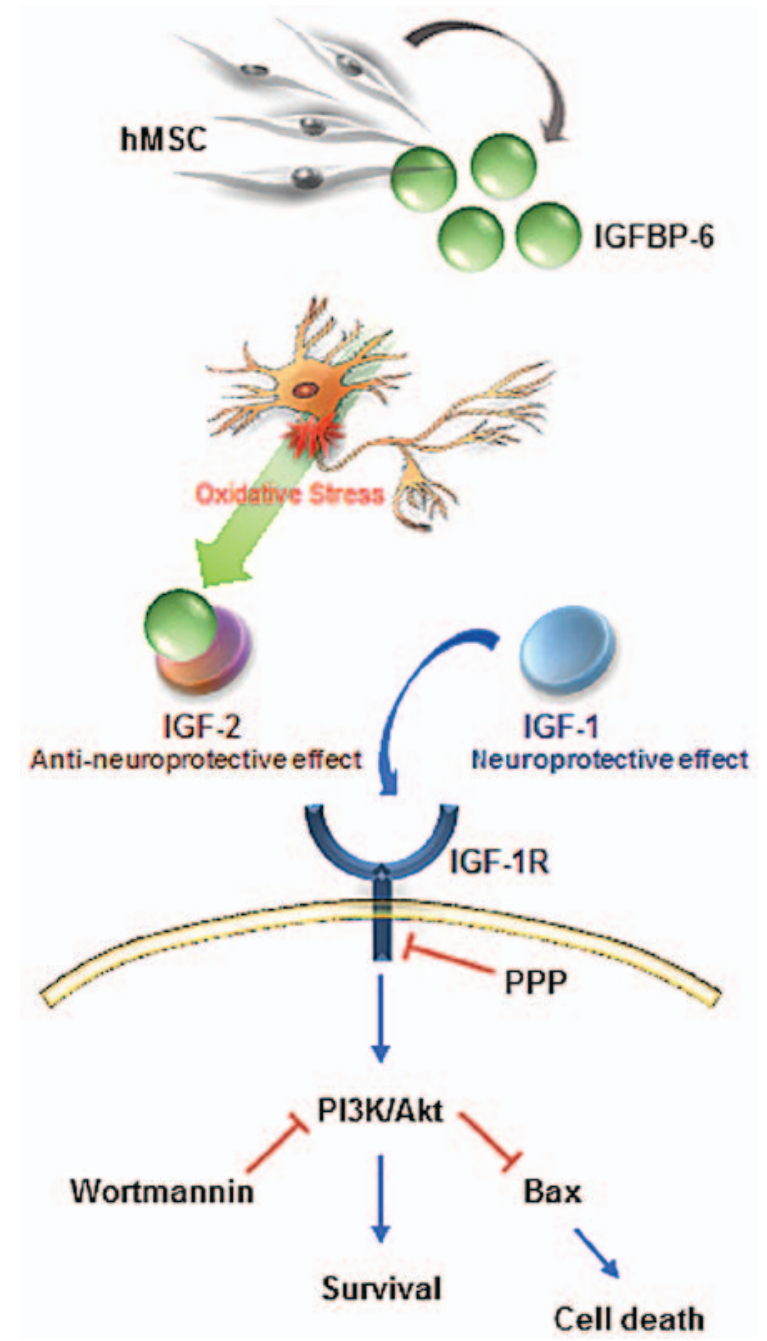

Figure 6. Diagram of the molecular mechanisms underlying the neuroprotective effect of IGFBP-6 via IGF-1R-dependent signaling. IGFBP-6 released from hMSCs prevented neuronal death induced by oxidative stress via the IGF-1R-mediated activation of Akt. IGFBP-6 inhibited the translocation of Bax to the mitochondria via the activation of PI3K/Akt, suggesting a potential role of IGFBP-6 in neuroprotection against oxidative stress through the IGF-1R pathway. hMSC, human mesenchymal stem cell; IGF-1R, insulin-like growth factor-1 receptor; IGFBP-6 Ab, insulin-like growth factor binding protein-6 antibody; Bax, B-cell lymphoma 2-like protein 4; PI3K, phosphoinositide 3-kinase; PPP, picropodophyllin.

Effect of hMSC transplantation in an ex vivo model of spinal cord injury. In our previous study, it was demonstrated that the transplantation of hMSCs significantly increased cell survival in LPC-treated demyelinated organotypic spinal cord slice cultures (15). Demyelination is a pathological feature of several neurological disorders and is caused by oxidative stress in neuroinflammation (20). The present study used an ex vivo model of spinal cord injury to examine whether hMSCs exert their neuroprotective role through IGFBP-6. Demyelination by LPC treatment notably increased the average number of TUNEL-stained cells per slice, compared with that in the untreated control, whereas transplantation of hMSCs significantly decreased the average number of TUNEL-stained cells per slice by $31 \pm 5.5 \%$, compared with that in the LPC-treated slices $(\mathrm{P}<0.01)$ (Fig. 5). In addition, pre-incubation of the LPC-treated slices with anti-IGFBP-6 antibody resulted in a marked reversal of the anti-apoptotic effect of hMSC transplantation. Anti-IGFBP-6 antibody treatment in the hMSC-transplanted slices increased the average number of TUNEL-stained cells per slice, compared with that of the LPC-treated slices. These results indicated that IGFBP-6 was critical for hMSC-mediated cell survival in the demyelinated organotypic spinal cord slice cultures. Taken together, these results suggested that IGFBP-6 was important in neuronal survival through activation of the Akt- and IGF-1R-mediated signaling pathway (Fig. 6).

\section{Discussion}

The therapeutic effects of hMSCs have been attributed to their multipotency to replace damaged or lost cells and the secretion of paracrine factors (4). hMSCs promote neuronal survival and neuritogenesis by secreting neurotrophic factors (21). hMSC-CM can increase neuronal survival and neurite outgrowth, which is associated with higher levels of secreted IGF-1, HGF, VEGF and TGF- $\beta$ (22). However, the detailed function of each paracrine factor in $\mathrm{hMSC}-\mathrm{CM}$ remains to be fully elucidated. IGFBP-6, one of the abundant growth factors released from hMSCs, increases lifespan and decreases apoptosis (23). It also has direct mitogenic and anti-apoptotic effects in Saos-2/B-10 cells, a human osteoblastic osteosarcoma cell line (24).

The present study demonstrated for the first time, to the best of our knowledge, that IGFBP- 6 released from hMSCs possessed neuroprotective effects in a primary cortical neuron culture. IGFBP-6 in hMSC-CM protected primary cortical neurons against the oxidative stress induced by $\mathrm{H}_{2} \mathrm{O}_{2}$. The phosphorylation of Akt was markedly enhanced in damaged primary cortical neurons treated with hMSC-CM, compared with that in cells without hMSC-CM treatment, and the expression of pAkt inhibited neuronal cell death. Additionally, the activation of Akt was reduced following the addition of anti-IGFBP-6 inhibitory antibody in the hMSC-CM, suggesting that IGFBP-6 regulated the level of pAkt. It has been reported that the activation of Akt in neurons prevents the translocation of pro-apoptotic Bax to the mitochondria by inhibiting p53-mediated transactivation $(25,26)$. The results of the present study also demonstrated that hMSC-CM treatment prevented the translocation of Bax to the mitochondria induced by $\mathrm{H}_{2} \mathrm{O}_{2}$ treatment. However, incubation with the anti-IGFBP-6 antibody reversed the effect of hMSC-CM treatment and increased the level of mitochondrial Bax following $\mathrm{H}_{2} \mathrm{O}_{2}$ treatment.

Consistent with the results of the present study, several studies have shown a specific role of IGFBP-6 in cell survival. IGFBP-6 inhibits the senescence and death of human fibroblasts (23), and IGFBP-6 was found to be markedly upregulated in the brain following hypoxic-ischemic (HI) injury, whereas IGF-2 was not (27). Another study reported that treatment with IGF-2 increased neuronal loss in the hippocampus and dentate gyrus during brain damage caused by HI injury, and inhibited IGF-1-induced neuroprotection (28). As IGFBP-6 inhibits IGF-2 due to its high binding affinity (Fig. 4) (9), the neuroprotective effects of IGFBP-6 can be potentiated by free IGF-1 (27). IGF-1 regulates DNA synthesis, cell growth and anti-apoptotic pathways, and induces neuronal differentiation (29). In general, IGF-1 and IGF-2 bind to IGF-1R, 
thereby inducing the activation of the Akt and/or ERK pathways $(16,30)$. The results of the present study suggested that IGFBP-6 primarily activated Akt, rather than ERK (Fig. 1B). In addition, the significantly increased expression of pAkt following hMSC-CM treatment was reversed by PPP treatment (Fig. 2B), indicating that IGFBP-6 may enhance the phosphorylation of Akt through IGF-1R-mediated signaling. These findings suggested that IGFBP-6 inhibited the apoptotic effect of IGF-2 and regulated IGF-1R-mediated signaling via free IGF-1, which activated the anti-apoptotic PI3K/Akt signaling pathway.

The IGF-independent effects of IGFBP-6 (6) may also contribute to neuroprotection in primary cortical neurons. Gli1, a full length transcriptional activator of hedgehog signaling, maintains cell survival by facilitating the transcription of Bcl-2 genes through binding to promoter regions in IGFBP-6 $(31,32)$ and mediates the survival of diffuse large B-cell lymphoma cells by promoting the transcription of Akt (33). This is consistent with the finding of the present study that hMSC-CM containing IGFBP-6 increased the level of pAkt.

However, the function of IGFBP-6 remains controversial. Several studies have reported on the anti-proliferative activity of IGFBP-6. For example, IGFBP-6 predominantly binds to IGF-2 and inhibits IGF/insulin signaling to suppress cell proliferation and survival in various cancer cell lines $(9,34)$. The present study provided insight into IGFBP-6-mediated cellular survival mechanisms in the nervous system. However, further investigations are required, particularly regarding the detailed mechanisms of IGF-1 and IGF-1R signaling through IGFBP-6. Taken together, the results of the present study indicated that IGFBP-6 is an important neuronal survival factor secreted from hMSCs, suggesting that IGFBP-6 is a desirable factor for the treatment of neurodegenerative diseases.

\section{Acknowledgements}

This study was supported by the Basic Science Research Program through the National Research Foundation of Korea (grant. no. M-SC, NRF-2015R1D1A1A01056950) funded by the Ministry of Education, and by a grant from the Korean Health Technology R\&D Project (grant no. M-SC, A120476) of the Ministry of Health and Welfare, Republic of Korea.

\section{References}

1. Reynolds A, Laurie C, Mosley RL and Gendelman HE: Oxidative stress and the pathogenesis of neurodegenerative disorders. Int Rev Neurobiol 82: 297-325, 2007.

2. Uttara B, Singh AV, Zamboni P and Mahajan RT: Oxidative stress and neurodegenerative diseases: A review of upstream and downstream antioxidant therapeutic options. Curr Neuropharmacol 7 : 65-74, 2009.

3. Uccelli A, Moretta L and Pistoia V: Mesenchymal stem cells in health and disease. Nat Rev Immunol 8: 726-736, 2008.

4. Mezey E: The therapeutic potential of bone marrow-derived stromal cells. J Cell Biochem 112: 2683-2687, 2011.

5. Park HW, Lim MJ, Jung H, Lee SP, Paik KS and Chang MS: Human mesenchymal stem cell-derived Schwann cell-like cells exhibit neurotrophic effects, via distinct growth factor production, in a model of spinal cord injury. Glia 58: 1118-1132, 2010.

6. Firth SM and Baxter RC: Cellular actions of the insulin-like growth factor binding proteins. Endocr Rev 23: 824-854, 2002.
7. Hwa V, Oh Y and Rosenfeld RG: The insulin-like growth factorbinding protein (IGFBP) superfamily. Endocr Rev 20: 761-787, 1999.

8. Hoyt KR, Gallagher AJ, Hastings TG and Reynolds IJ: Characterization of hydrogen peroxide toxicity in cultured rat forebrain neurons. Neurochem Res 22: 333-340, 1997.

9. Bach LA: Recent insights into the actions of IGFBP-6. J Cell Commun Signal 9: 189-200, 2015.

10. Oskowitz A, McFerrin H, Gutschow M, Carter ML and Pochampally R: Serum-deprived human multipotent mesenchymal stromal cells (MSCs) are highly angiogenic. Stem Cell Res 6: 215-225, 2011.

11. Koike H, Ito K, Takezawa Y, Oyama T, Yamanaka H and Suzuki K: Insulin-like growth factor binding protein-6 inhibits prostate cancer cell proliferation: Implication for anticancer effect of diethylstilbestrol in hormone refractory prostate cancer. Br J Cancer 92: 1538-1544, 2005.

12. Cohen DM: Urea-inducible Egr-1 transcription in renal inner medullary collecting duct (mIMCD3) cells is mediated by extracellular signal-regulated kinase activation. Proc Natl Acad Sci USA 93: 11242-11247, 1996.

13. Wu EH and Wong YH: Involvement of Gi/o proteins in nerve growth factor-stimulated phosphorylation and degradation of tuberin in PC-12 cells and cortical neurons. Mol Pharmacol 67: 1195-1205, 2005

14. Wahane SD, Hellbach N, Prentzell MT, Weise SC, Vezzali R, Kreutz C, Timmer J, Krieglstein K, Thedieck K and Vogel T: PI3K-p110-alpha-subtype signalling mediates survival, proliferation and neurogenesis of cortical progenitor cells via activation of mTORC2. J Neurochem 130: 255-267, 2014.

15. Cho JS, Park H-W, Park S-K, Roh S, Kang SK, Paik KS and Chang MS: Transplantation of mesenchymal stem cells enhances axonal outgrowth and cell survival in an organotypic spinal cord slice culture. Neurosci Lett 454: 43-48, 2009.

16. Saltiel AR and Kahn CR: Insulin signalling and the regulation of glucose and lipid metabolism. Nature 414: 799-806, 2001.

17. Pang Y, Zheng B, Fan LW, Rhodes PG and Cai Z: IGF-1 protects oligodendrocyte progenitors against TNFalpha-induced damage by activation of PI3K/Akt and interruption of the mitochondrial apoptotic pathway. Glia 55: 1099-1107, 2007.

18. Wolter KG, Hsu Y-T, Smith CL, Nechushtan A, Xi XG and Youle RJ: Movement of Bax from the cytosol to mitochondria during apoptosis. J Cell Biol 139: 1281-1292, 1997.

19. Hsu YT, Wolter KG and Youle RJ: Cytosol-to-membrane redistribution of Bax and Bcl-X(L) during apoptosis. Proc Natl Acad Sci USA 94: 3668-3672, 1997.

20. Ljubisavljevic S: Oxidative stress and neurobiology of demyelination. Mol Neurobiol 53: 744-758, 2016.

21. Crigler L, Robey RC, Asawachaicharn A, Gaupp D and Phinney DG: Human mesenchymal stem cell subpopulations express a variety of neuro-regulatory molecules and promote neuronal cell survival and neuritogenesis. Exp Neurol 198: 54-64, 2006.

22. Nakano N, Nakai Y, Seo T-B, Yamada Y, Ohno T, Yamanaka A, Nagai Y,Fukushima M,Suzuki Y,Nakatani T, et al: Characterization of conditioned medium of cultured bone marrow stromal cells. Neurosci Lett 483: 57-61, 2010.

23. Micutkova L, Diener T, Li C, Rogowska-Wrzesinska A, Mueck C, Huetter E, Weinberger B, Grubeck-Loebenstein B, Roepstorff P, Zeng $\mathrm{R}$, et al: Insulin-like growth factor binding protein- 6 delays replicative senescence of human fibroblasts. Mech Ageing Dev 132: 468-479, 2011.

24. Schmid C, Keller C, Gosteli-Peter M and Zapf J: Mitogenic and antiapoptotic effects of insulin-like growth factor binding protein-6 in the human osteoblastic osteosarcoma cell line Saos-2/B-10. Biochem Biophys Res Commun 263: 786-789, 1999.

25. Brunet A, Datta SR and Greenberg ME: Transcription-dependent and -independent control of neuronal survival by the PI3K-Akt signaling pathway. Curr Opin Neurobiol 11: 297-305, 2001.

26. Yamaguchi A, Tamatani M, Matsuzaki H, Namikawa $K$, Kiyama H, Vitek MP, Mitsuda N and Tohyama M: Akt activation protects hippocampal neurons from apoptosis by inhibiting transcriptional activity of p53. J Biol Chem 276: 5256-5264, 2001.

27. Beilharz EJ, Russo VC, Butler G, Baker NL, Connor B, Sirimanne ES, Dragunow M, Werther GA, Gluckman PD, Williams CE, et al: Co-ordinated and cellular specific induction of the components of the IGF/IGFBP axis in the rat brain following hypoxic-ischemic injury. Brain Res Mol Brain Res 59: 119-134, 1998. 
28. Guan J, Williams CE, Skinner SJ, Mallard EC and Gluckman PD The effects of insulin-like growth factor (IGF)-1, IGF-2, and des-IGF-1 on neuronal loss after hypoxic-ischemic brain injury in adult rats: Evidence for a role for IGF binding proteins. Endocrinology 137: 893-898, 1996.

29. Chesik D, De Keyser J and Wilczak N: Insulin-like growth factor binding protein-2 as a regulator of IGF actions in CNS: Implications in multiple sclerosis. Cytokine Growth Factor Rev 18: 267-278, 2007.

30. LeRoith D, Werner H, Beitner-Johnson D and Roberts CT Jr: Molecular and cellular aspects of the insulin-like growth factor I receptor. Endocr Rev 16: 143-163, 1995.

31. Yoon JW, Kita Y, Frank DJ, Majewski RR, Konicek BA, Nobrega MA, Jacob H, Walterhouse D, Iannaccone P: Gene expression profiling leads to identification of GLI1 binding elements in target genes and a role for multiple downstream pathways in GLI1 induced cell transformation. J Biol Chem 277: 5548-5555, 2002.
32. Xu XF, Guo C-Y, Liu J, Yang WJ, Xia YJ, Xu L, Yu YC and Wang XP: Gli1 maintains cell survival by up-regulating IGFBP6 and Bcl-2 through promoter regions in parallel manner in pancreatic cancer cells. J Carcinog 8: 13, 2009.

33. Agarwal NK, Qu C, Kunkalla K, Liu Y and Vega F: Transcriptional regulation of serine/threonine protein kinase (AKT) genes by glioma-associated oncogene homolog 1 . J Biol Chem 288: 15390-15401, 2013.

34. Duan C and Xu Q: Roles of insulin-like growth factor (IGF) binding proteins in regulating IGF actions. Gen Comp Endocrinol 142: 44-52, 2005.

(i) $(5)$ This work is licensed under a Creative Commons Attribution-NonCommercial-NoDerivatives 4.0 International (CC BY-NC-ND 4.0) License. 